\title{
Discrepancies in Health Care Funding for Individuals with Special Needs
}

\author{
(See article by Lijing Ouyang et al.)
}

\author{
William O. Walker, Jr.* \\ Professor of Pediatrics, University of Washington School of Medicine, WA, USA \\ Chief, Division of Developmental Medicine, Seattle Children's Hospital, Seattle, WA, USA
}

\begin{abstract}
Approximately 47 million Americans - $16 \%$ of the total US population - lack health insurance [10]. An additional 25 million have insurance plans that limit their access to services, or cover only a small percentage of expenses - they, in fact, are underinsured. In 1997, the original State Children's Health Insurance Program (SCHIP) established coverage for children who did not qualify for Medicaid but whose families could not afford private insurance. This legislation reduced the rate of uninsured low-income children by almost one third, from $23 \%$ ( 7.6 million children) to $14 \%$ (5.6 million children) and improved health care quality. The prevalence of uninsured children in 2007 ranged from $4.1 \%$ to $21.2 \%$ across various states [7]. In 2009, 7 million children were enrolled in SCHIP and 29 million children were enrolled in Medicaid. CHIP's (Children's Health Insurance Program) reauthorization in 2009 liberalized the means test criteria $(>300 \%$ of the federal poverty level) of the initial legislation, extended coverage to more recent immigrants (less than 5 years) and authorized the states to cover an increased number of services, including dental and mental health care.

Children under the age of 18 years comprise $26 \%$ of the US population, yet they are responsible for only $10 \%$ of total healthcare expenditures and consume only $9 \%$ of public funds for healthcare [10]. However, the $14 \%$ of children and youth in the United States with special health care needs account for $42 \%$ of the medi-
\end{abstract}

\footnotetext{
* Address for correspondence: William O. Walker, Jr. MD, Division of Genetics and Developmental Medicine, Seattle Children's Hospital (M/S A-7938), 4800 Sandpoint Way NE, Seattle, WA 98105, USA. Tel.: +1 2069873664 ; Fax: +1 2069873824 ; E-mail: william. walker@seattlechildrens.org.
}

cal expenditures in their age group. The Maternal Child Health Bureau's defines CYSHCN (children and youth with special health care needs) as "those who have or are at risk for a chronic physical, developmental, behavioral, or emotional condition and who also require health and related services of a type or amount beyond that required by children generally" [1]. Higher survival rates for CYSHCN are reported over the past three decades across a wide range of chronic medical conditions; $90 \%$ will reach their 20th birthday [8]. A study by Callahan et al. showed $11.4 \%$ of 16 - to 25 -year-olds in the United States reported having a disability -4 million young adults. [11]

Ouyang et al. describe in detail the financial costs of obtaining necessary medical care for specific medical conditions/symptoms across the life span for children and adults with spina bifida [5]. While earlier studies have documented significantly higher medical costs for both children and adults with spina bifida, the authors' unique contribution is in describing the relative frequencies of medical conditions and new sources of morbidity and mortality that advancing age, related to longer survival, introduces. Their analysis of data from a large insurance claims database is an important step toward better understanding of and planning for changing ambulatory medical needs. In defining their reasons for selecting this specific database (MarketScan Commercial Claims and Encounters Database) for analysis, the authors identified several potential sources of health care and health care financing disparity in the spina bifida population: private vs. public insurance; fee for service vs. capitated plans; primary vs. specialty care. Because this database only includes private claims data, their hope was that the impact of some of these fac- 
tors would be minimized. Their findings emphasize the lifetime need for medical care and raise the issues of access to and appropriateness of that care for individuals with spina bifida. Similar factors contributing to discrepancies in health care should be expected in other chronic conditions. Claims based, administrative database analysis, however, can only evaluate the care obtained and not the care required. The authors cannot address how costs are distributed between insurance (covered) and the individual (uncovered) and how the burden from these additional costs affect the individual with spina bifida access to appropriate and necessary care.

Over $50 \%$ of Ouyang's study sample were dependents (children and adults) of the primary insurance beneficiary. Their ability to obtain appropriate medical care would likely be very different if that relationship, and its associated insurance benefits, did not exist. Those familiar with the details of recently passed health reform legislation suggest that several aspects of that legislation may alter insurance coverage for individuals with special health care needs. Specifically, it may become economically advantageous for employers to significantly limit or even eliminate family coverage from their future insurance plans, adversely affecting insurance coverage, medical access and necessary health care for previously covered dependents. High-risk insurance pools and decreased Medicaid reimbursement rates may have similar adverse effects. These changes would further contribute to the existing disparities in health care access and financing for individuals with special needs.

Numerous studies and surveys have assessed having health insurance and its positive influence on access, utilization, satisfaction, quality, and health outcomes for CYSHCN [6]. The strongest evidence supports the positive effects of having insurance on access and utilization [1]. A lack of insurance is the strongest indicator of delayed or foregone care for CYSHCN and increases their likelihood of experiencing barriers to health care. However, the evidence is less conclusive for satisfaction with care and quality of care. No studies have effectively assessed the long-term impact of having or not having health insurance: long-term child development, family stress, quality of life, and longterm economic burden on the community. The authors provide some initial data evaluating the life-span impact of specific symptoms including pain and sleep disturbance on individuals with spina bifida. A logical next step would be to consider the World Health Organization (WHO) International Classification of Func- tion's (ICF) concepts of activity and participation as important and valuable frameworks for future health insurance outcome studies.

The authors demonstrated that the significant difference between annual per capita health care costs in persons with spina bifida compared to the general population continues into adulthood. The high per event cost associated with relatively infrequent conditions (diseases of the nervous system - compression of brain) as well as the high total cost associated with more frequent diagnoses such as urinary tract infection and headache will require careful consideration in developing different models of future health care coverage and financing. The type of coverage and payment mechanism relates to quality of care. There is little difference in utilization rates (including subspecialty care) between CYSHCN with private and public coverage while payment mechanism (fee-for-service or managed care) has been shown to affect utilization. In the past decade, major changes have occurred in children's health insurance, including a shift from private insurance to public coverage (CHIP), as well as an acceleration of the trend toward managed care as the predominant financing and delivery mechanism under both private and public plans. Once the most common form of coverage, indemnity plans accounted for $73 \%$ of employment based health insurance as late as 1988 [4]. By 2007 , only $3 \%$ of workers with employer coverage were enrolled in indemnity plans, whereas $57 \%$ were enrolled in preferred provider organizations (PPOs), $21 \%$ in health maintenance organizations (HMOs), and $13 \%$ in point-of-service plans, which combine features of HMOs and Preferred Provider Organizations (PPOs). Several recent studies showed that CYSHCN enrolled in managed care fared better than their fee-for-service peers did on access indicators.

There have been other efforts to better integrate the principles of managed care into services for CYSHCN. Unfortunately, care models that may work well for adults are not guaranteed to work equally well for children, and especially CYSHCN. This is especially true when children are considered "little adults". The needs of CYHSCN with disabilities differ from those of adults with disabilities, even in a managed care environment. Their health and development depend greatly on their families' health and socioeconomic status. Development is a critical part of a child's overall health. The needs and expected outcomes of children vary across different developmental stages. Illness and disability can delay, sometimes irreversibly, a child's normal development. The most significant difference and the one 
that will likely have the greatest impact on health care planning is the different epidemiology and prevalence of disabilities between these two populations. Children have many rare or low-incidence conditions and few common ones. In adults, there are few rare conditions and several common ones. These inherent differences will need to be considered as CYSHCN survive longer and change the composition and characteristics of the population of adults with disabilities [12]. By describing the needs and relative costs of medical care for individuals with spina bifida over their life span, Ouyang provide the baseline data for future interventions to assess comparative effectiveness.

While discussions regarding health care often focus on health insurance status, having health insurance is not a simple "have" or "don't have" question. There is neither a single accepted definition for "underinsured" nor a standard methodology to determine specific rates. The common definition elements include health care insurance that:

- requires excessive out-of-pocket expenditures

- has significant limits with respect to what health care services are covered

- fails to cover health care expenses perceived by the insured person to be essential for his or her health.

Perception of and satisfaction with the health care received and the ability to pay for health care (insurance premiums, copayments, deductibles) are additional considerations in defining "underinsurance". Recent efforts at assessing the rate of underinsurance in children have attempted to utilize more comprehensive methods that avoid arbitrary out of pocket thresholds or benefit level benchmarks that have been used in adult studies; these methods likely underestimate the true significance of "underinsurance" and its effect on comprehensive health care access [6].

The risk for being underinsured is affected by a number of factors: race/ethnicity, child's age, poverty level, where a child lives, and the degree to which the child's activities are affected by the condition [3]. Children with private insurance are twice as likely to be underinsured as children with public insurance [6]. Nationally, an estimated one third of CYSHCN are underinsured [7]. An analysis of data from the 2007 National Survey of Children's Health demonstrated that the number of underinsured children exceeded the number of children without insurance, that CYSHCN were more likely to be underinsured and that inadequate coverage of charges was the most common reason [6]. The source of underinsurance varies by type of cover- age, with inadequate coverage of needed services and providers being the primary source of underinsurance for publicly insured CYSHCN and inadequate coverage of a reasonable amount of costs being the primary source of underinsurance for privately insured CYSHCN.

Underinsurance is associated with lack of a medical home, delayed or omitted care and having difficulty obtaining referrals [6]. Strengthening primary care, both as a health systems orientation and as a set of functions delivered by a usual source of care, is the best way to address these gaps and to improve quality, outcomes, and cost of care for individuals with special health care needs. The recently passed Patient Protection and Affordable Care Act of 2010 incorporates several provisions intended to strengthen primary care [2]. These provisions include expanding the primary care workforce, equipping primary care practitioners with new capabilities, and reorienting the current delivery system through payment and organizational reforms to improve access to care. Hopefully, these changes will address specific issues for CYSHCN found to improve health care quality and access. Appropriate subspecialty care, the training and development of adult medical providers familiar with the natural history of various "childhood" disorders, and a transition from an "illness" care system to a "health" care system must be established as well.

\section{References}

[1] A.E. Jeffrey and P.W. Newacheck, Role of Insurance for Children With Special Health Care Needs: A Synthesis of the Evidence, Pediatrics 118 (2006), e1027-e1038.

[2] J.S. Palfrey and R. Hall, Health Care Reform: The Doorway to Health Care Transformation, Pediatrics 126 (2010), 374-375.

[3] L. Honberg, M. McPherson, B. Strickland, J.C. Gage and P.W. Newacheck, Assuring Adequate Health Insurance: Results of the National Survey of Children With Special Health Care Needs, Pediatrics 115 (2005), 1233-1239.

[4] L.E. Honberg, M.D. Kogan, D. Allen, B.B. Strickland and P.W. Newacheck, Progress in Ensuring Adequate Health Insurance for Children With Special Health Care Needs, Pediatrics 124 (2009), 1273-1280.

[5] L. Ouyang, S.D. Grosse, J. Thibadeau, M. Swanson and V.A. Campbell, Outpatient Medical Conditions Among Children and Adults with Spina Bifida in the United States: Frequency and Expenditures, Journal of Pediatric Rehabilitation Medicine 3(2010), $\mathrm{xx}-\mathrm{xx}$.

[6] M.D. Kogan, P.W. Newacheck, S.J. Blumberg, R.M. Ghandour, G.K. Singh, B.B. Strickland and P.C. van Dyck, Underinsurance among Children in the United States, New England Journal of Medicine 363 (2010), 841-851. 
[7] M.D. Kogan, P.W. Newacheck, S.J. Blumberg, K.M. Heyman, B.S. Strickland, G.K. Singh and M.B. Zeni, State Variation in Underinsurance Among Children With Special Health Care Needs in the United States, Pediatrics 125 (2010), 673-680.

[8] N.G. Peter, C.M. Forke, K.R. Ginsburg and D.F. Schwarz. Transition From Pediatric to Adult Care: Internists' Perspectives, Pediatrics 123 (2009), 417-423.

[9] P.W. Newacheck, A.J. Houtrow, D.L. Romm, K.A. Kuklthau, S.R. Bloom, J.M. Van Cleave and J.M. Perrin, The Future of Health Insurance for Children With Special Health Care
Needs, Pediatrics 123 (2009), e940-e947.

[10] R. Stein and M. Silverstein, Potential implications of US healthcare reform for American children, Arch Dis Child $\mathbf{9 5}$ (2010), 578-579.

[11] S.T. Callahan and W.O. Cooper, Continuity of Health Insurance Coverage Among Young Adults With Disabilities, Pediatrics 119 (2007), 1175-1180.

[12] T.A. Kastner and the Committee on Children With Disabilities, Managed Care and Children With Special Health Care Needs, Pediatrics 114 (2004), 1693-1698. 